\title{
PUBLICACIONES DE PRINCIPIOS DEL SIGLO XX SOBRE LAS MINAS DE ORO EN COSTA RICA
}

\author{
Siegfried Kussmaul \\ Escuela Centroamericana de Geología, Apdo. 214.2060, Costa Rica \\ skussmau@geologia.ucr.ac.cr
}

(Recibido: 20/07/06; aceptado: 09/02/07)

\begin{abstract}
Three articles about the gold mines of Costa Rica, published by international mining journals at the beginning of the 20th century, are analyzed. The objective of two of the articles is to attract the interest in the investment of foreign capital for the development of the gold mines. The articles were written during the "Second Cycle of Gold Mining in Costa Rica", when numerous mining engineers carried out evaluations of the older gold mines. The data presented seem to be exaggerated, especially with respect to the width and tenor of the veins.

Keywords: Gold mines, Costa Rica, Aguacate district, Montes de Oro district, Abangares district.

RESUMEN: Se comentan tres artículos sobre las minas de oro en Costa Rica, publicados a principios del siglo XX en revistas internacionales especializadas. De los tres artículos, dos son más bien promociones de la riqueza minera en Costa Rica. Fueron escritos durante el "Segundo Ciclo Minero de Costa Rica" caracterizado por la llegada de varios ingenieros de minas extranjeros que realizaron evaluaciones de las minas antiguas. Los datos que ellos aportan son hoy en día difíciles de creer. Por ejemplo, mencionan espesores enormes de las vetas y leyes de oro sumamente altas.

Palabras clave: Minas de oro, Costa Rica, Distrito de Aguacate, Montes de Oro, Abangares.
\end{abstract}




\section{INTRODUCCIÓN}

En esta sección se presentan tres artículos en idioma inglés que fueron publicados a principios del siglo XX en tres diferentes revistas de minería que todavía hoy en día tienen una divulgación muy amplia: Un artículo de Milton Franklin Reitz (1902), titulado "The gold mines of Costa Rica" en The Engineering and Mining Journal (fundado en 1866); "Geology and development of Aguacate mines, Costa Rica" por R.A. Crespi (1907) en The Mining World, y "Mineral resources of Costa Rica" en The Mining Journal (revista internacional fundada en 1835). Por su antigüedad, estas publicaciones son poco conocidas en Costa Rica, por lo cual consideramos oportuno reproducirlas en este volumen especial. Los artículos originales contienen errores tipográficos, en especial en lo que se refiere a los nombres geográficos, los cuales no se corrigieron.

En este contexto, se debe mencionar también al artículo clásico sobre la minería del oro en Costa Rica, titulado "Las Minas del Monte del Aguacate y Los Castros", escrito por el Dr. Ernesto Melliss, traducido al español por Manuel Carazo Peralta y publicado en el año 1891. Es probablemente la mejor publicación sobre las minas de oro en Costa Rica.

Muchos se preguntarán por qué unas de las primeras publicaciones geológicas de Costa Rica tratan de la minería del oro. La explicación es que, durante siglos el oro era objeto de culto y adorno antes de ser usado como moneda o base de referencia para el intercambio de mercancías y parte de las reservas monetarias de los países. En 1824 el gobierno de Costa Rica autorizó el acuñamiento de oro, y las primeras monedas en oro y cobre fueron acuñadas en el año 1828 en los Horcones, cerca del río Grande. El patrón oro se usó a lo largo de todo el siglo XIX y la primera mitad del siglo XX. Con la introducción del patrón oro, las monedas de oro pasaron cada vez con menos frecuencia de mano en mano, de modo que el oro se quedó solamente en las bodegas de los bancos como garantía del papel moneda. Recién a finales del siglo pasado el oro adquirió también gran importancia en varias ramas de la industria.
Ningún otro mineral u objeto despertó tal veneración como el oro. Ya en el siglo V a.C., el poeta griego Píndaro describió el oro como "hijo de Zeus, al que no devoran ni la polilla ni el herrumbre, pero cuya suprema posesión devora la mente del hombre" (en Bernstein, 2000). Una caracterización más moderna la dio $\mathrm{H}$. Hoover, quien dijo en 1933 al presidente electo de los Estados Unidos de América, Roosevelt: "Tenemos oro porque no podemos confiar en los gobiernos" o la del presidente francés De Gaulle, quien dijo en 1965 que "El oro carece de nacionalidad, es eterno y universalmente aceptado como la inversión fiduciario inalterable por excelencia." De Gaulle convirtió las reservas francesas de dólares en oro y provocó una crisis financiera mundial, que finalmente obligó al presidente Nixon en el año 1971 a suspender la convertibilidad del dólar en oro.

\section{DATOS BIOGRÁFICOS DE LOS AUTORES}

\section{Milton Franklin Reitz}

Sobre su biografía no se conoce mucho. Como él mismo menciona en su artículo, estuvo dos años en Costa Rica trabajando como ingeniero de minas en la evaluación minera en los montes del Aguacate y visitando muchas minas en Costa Rica.

\section{Roberto A. Crespi Guillon}

Ingeniero y empresario minero, muy conocido a finales del siglo XIX y principios del siglo XX. En 1893 adquirió la mina Tres Amigos en Abangares por la suma de 1000 pesos. El año siguiente formó una sociedad con el nombre de "Compañía de la Mina Tres Amigos", integrado por él y los señores Minor C. Keith y Carlos Volio T., con un capital de 100000 dólares (Cabrera, 1924). En 1900 vendieron la mina a la "Abangares Gold Fields of Costa Rica" en la suma de 5000 pesos. La compañía R.A. Crespi \& Co. compró en el año 1899 también la mina Macacona (Reitz, 1902; Anónimo, 1914). 
En el artículo "Mineral Resources of Costa Rica" del año 1914, el Sr. Crespi es mencionado como el gerente y uno de los principales accionistas de la "Costa Rica Exploration Company" y como un fuerte impulsor de la minería de oro en Costa Rica.

La mejor reseña laudatoria al señor Crespi la escribió Victor Manuel Cabrera (1924), encargado de la recopilación de los documentos relativos a la incorporación del Partido de Nicoya a Costa Rica. En el capítulo "Las Regiones Mineras" dice:

"Las Minas de Abangares constituyen por sí solas el más soberbio alarde de lo que es capaz de conseguir la voluntad de un hombre tenaz en sus propósitos y tendiendo a su disposición el campo de actividades que forman el carácter norteamericano; pues aquel pequeño estado dentro del Estado, con su movimiento de centenares de empleados y de braceros, con sus colosales instalaciones mecánicas, su planta eléctrica, su inmensa red de alambres conductores de energía, sus 22 kilómetros de línea telefónica, su funicular de vagonetas, su ferrocarril de vía estrecha, sus grandes turbinas, su comisariato de víveres, sus almacenes de piezas de repuesto, sus escuelas, sus caminos y puertos, su fábrica de hielo, la oficina de ensayos y laboratorios, todo y cada uno de esos detalles que forman el grandioso conjunto de la instalación minera, que puede citarse como un acabado modelo en el género, tiene por fuerza impulsora la constancia y el espíritu de un hombre: Mr. Roberto Crespi, quien supo interesar en esta explotación un capital de consideración."

Hoy en día esas palabras no llaman mucho la atención, pero tenemos que considerar que se habla de un desarrollo que se llevó a cabo hace más de cien años.

El tercer artículo, "Mineral resources of Costa Rica" es un resumen de publicaciones, principalmente de la Revista Económica. No se conoce donde ni quién publicó la revista económica. Reitz (1902) apunta en su artículo que en las revistas de los Estados Unidos se encuentran frecuentemente resúmenes sobre distintos distritos mineros de todo el mundo. Algunas de estas publicaciones son cuestionables, ya que los autores gastan poco tiempo en su preparación, dejan al lado los datos exactos y prestan más importancia a las leyendas y tradiciones.

\section{LEYENDAS SOBRE EL ORO EN COSTA RICA}

En muchos documentos que fueron mandados a la corona española se menciona la pobreza de Costa Rica con respecto a minerales o la falta de minas de oro y de plata. Sin embargo, se habla de tesoros mineros en Costa Rica, al decir, con respecto a la fundación de la villa de Bruselas, en el Golfo de Nicoya: "Poblóla el año 1524 el capitán Francisco Hernández de Córdova en el Estrecho Dudoso, en el asiento de Urutina; y por una parte tenía la mar, por otra los llanos, y por tercera, la Sierra de las Minas" (Jinesta, 1938). La población de Bruselas estaba ubicada tierra adentro, frente a la isla de Chira, y la Sierra de las Minas corresponde por lo tanto a la cordillera de Tilarán, donde la primera mina de oro fue descubierto apenas a finales del siglo XIX. Es por eso, que no se puede descartar la posibilidad de que ya antes de la Conquista Española, los indígenas sacaran oro en esta cordillera.

Según las leyendas, la mina de oro más rica de Costa Rica era la de Tisingal (ver Denyer \& Soto, 2000 y Denyer, 2001). El artículo "Mineral Resources of Costa Rica" presenta una nueva variante sobre Tisingal. Sin citar fuentes, menciona que esta mina fue destruida por los indios y que ellos cambiaron el curso de los ríos, con el fin de que los españoles no encontraran la mina. Esta versión se basa tal vez en la descripción de Reitz (1902) quien menciona, que un ciudadano inglés con nombre Phillips encontró en los montes del Aguacate una bonanza muy rica. Intentó a adquirir la concesión pero los dueños no aceptaron su propuesta. Regresó a Inglaterra para buscar más fondos, pero antes llenó el acceso al túnel con las rocas que se excavaron, dejando algunas señas que solamente él podría reconocer. 


\section{LLEGADA DE EXTRANJEROS}

La minería de oro a gran escala se desarrolló en Costa Rica a partir de 1821 en los montes del Aguacate. Araya Pochet (1976) denomina la época entre 1821 y 1843 como "Primer Ciclo Minero de Costa Rica", tiempo en el cual se alcanzó prosperidad debido a la exportación del oro y el comienzo del comercio con el exterior, principalmente con Estados Unidos y Europa. La actividad minera llegó a ocupar en la década de 1830 de un $45 \%$ a un $48 \%$ del total de las exportaciones de la nueva nación Costa Rica. La circulación del dinero producido por las minas trasformó completamente la economía del país.

Durante el primer ciclo minero, las minas estaban en manos de costarricenses, muchos de ellos políticos o sus familiares, como Rafael Moya, Nicolás Ulloa y Rafael Gallegos, jefes de estado; Demetrio Yglesias, familiar del presidente Rafael Iglesias Castro; Francisco María Iglesias, vicepresidente de la República; los hijos del expresidente Julio Acosta García; pero también Rafael Francisco Osejo, Miguel Bonilla, los hermanos Oreamuno, José Joaquín Mora y muchos otros (Melliss, 1891; Reitz, 1902; García, 1984).

Costa Rica no contaba con ingenieros de minas ni con obreros familiarizados con los quehaceres en las minas, así que se estimuló la inmigración. En 1824 se le giraron 800 pesos a Francisco Echeverría, residente en Guanajuato (México), para que viniera a establecer el laborío y beneficio de mina (Jinesta, 1938). El decreto del 21 de julio de 1825, dictado en Guatemala, permitió a los extranjeros "dedicarse al laborío de minas, pudiendo adquirir la propiedad y dominio de las mismas, siempre que no sea por medio de denuncios. Se daba un año de término para explotarlas, pasado el cual sin realizar el trabajo, podían ser denunciadas otra vez" (Jinesta, 1938). Llegaron al país Mateo Urandurraga de España, John Gerard y Ricardo Trevithick de Inglaterra, Carlos Merz y Juan Barth de Alemania, Santiago Millet de Francia, y Domingo Mattei, Mateo Bertora, Carlos Volio y Angel Franceschi de Italia (Jinesta, 1938). Uno de los más famosos era el ingeniero de minas inglés, Trevithick, quien a principios del siglo XIX construyó en su país un carruaje de vapor, poste- riormente invirtió en minas en el Perú y en 1824 abandonó Perú por problemas políticos y trabajó durante varios años las minas del Aguacate.

Una vez agotado el oro cerca de la superficie, la explotación artesanal ya no era posible, las minas tenían que cerrar y así terminó el "Primer Ciclo Minero". En el año 1849 descubrieron los placeres de oro en California, el año siguiente en Australia y en 1897 en Alaska. La fiebre del oro se propagó probablemente hasta Costa Rica, donde se originan muchos anuncios en los periódicos europeos promocionando la inversión (ver Denyer, 2001). Empieza la evaluación de las viejas minas en los montes del Aguacate y la búsqueda de nuevos yacimientos. En 1857 se descubre la mina de Paires y en 1864 la de Ciruelitas, ambas en la cordillera de Tilarán. Comienza una segunda ola de ingreso de extranjeros, pero esta vez con muchos ingenieros de minas, contratados con el fin de evaluar el potencial de oro en las minas antiguas y de diseñar métodos de explotación y beneficio más adecuados. Algunos nombres son: Hugo Reck, Ernesto Melliss; M.F. Reitz (autor de uno de los artículos), J.W. Kerr, A.F. Holden, H.C. Holthoff, C.H. Colburn, E.C. Small, S.F. Shaw y W.M. Gabb. También llegaron trabajadores de Nicaragua, Honduras, Jamaica, Inglaterra, Alemania, Italia, Líbano y China (García, 1984). Esta segunda época de inmigración coincidió con la construcción del ferrocarril al Caribe entre los años de 1870 y 1890 , donde se emplearon muchos trabajadores extranjeros, como chinos, italianos y afrocaribeños.

La investigación intensa tuvo sus frutos en el descubrimiento de más reservas y nuevos yacimientos, y nuevamente empezó una actividad minera intensa, el "Segundo Ciclo Minero", aproximadamente entre los años 1890 y 1930. Se descubrieron las ricas minas cerca de Abangares, denunciados al principio por costarricenses, pero poco a poco las minas pasan a manos de compañías extranjeras, en especial a la "Abangares Gold Fields of Costa Rica", cuyo accionista principal era Minor C. Keith. Anecdóticamente, a finales del año 1911 se dio en Abangares la primera huelga obrera del país, causada por problemas con guardas y capataces negros provenientes de Jamaica (García, 1984) 


\section{LABOREO MINERO EN EL SIGLO XIX}

El Primer Ciclo Minero llegó a su fin porque las minas utilizaron una precaria tecnología y trabajaron con limitada inversión de capital. Los concesionarios eran principalmente costarricenses sin experiencia en la minería, la explotación de las vetas se llevó a cabo de manera rudimentaria, solo se sacaron los minerales cerca de la superficie y, al profundizarse, los pozos y túneles se inundaron con agua por lo cual terminaron de explotarlos. Para separar el oro se molía el material de la veta en un molinete o rastra, y el oro se recuperaba por amalgamación sobre planchas de cobre cubiertos con mercurio y posterior evaporación del mercurio en un horno. La desventaja de esta metodología era que raras veces se recuperó más del 30\% del oro presente en la veta. Es por eso que Reitz (1902) encontró valores muy altos de oro en las lamas o desechos de estas minas antiguas.

Los tres artículos mencionan molinos del tipo arrastra, molino artesanal conocido en Costa Rica como "molinete" o "rastra", cuando está impulsado por bueyes. A pesar que el término arrastra viene de la palabra español "arrastrar" y explica bien el mecanismo del molino, en Costa Rica no se utiliza este término. Se trata de una roca grande y plana, fijada en un palo vertical y girada por personas o animales para moler el material extraído de la mina. Las arrastras fueron introducidas en América por los españoles y posteriormente reemplazadas por los molinos de mazos.

Durante el Primer Ciclo Minero solamente se explotó el mineral, pero no se hicieron estudios geológicos y ninguna exploración sistemática. Además, no solamente faltó capital para la instalación de maquinaria moderna, como molinos y bombas de agua, sino que mucho más grave fue la falta de vías de acceso a las minas para transportar esta maquinaria. A principios del siglo XIX no hubo ni ferrocarril ni caminos para carretas de bueyes, así que era imposible transportar un mazo de acero que pesara entre 400 y $900 \mathrm{~kg}$ para construir un molino que consta de 10 ó 20 de estos mazos. Es por eso que en los artículos de Reitz (1902) y Crespi (1907) se pone mucho énfasis a la cercanía de los yacimientos de la línea de ferrocarril o de caminos para carretas.
En la segunda mitad del siglo XIX ya se contó con un camino de carretas que comunicó Puntarenas con el Valle Central y permitió la movilización de maquinaria pesada. Existía todavía la obsesión por el oro y finalmente también las bases para transportar maquinaria pesada, así que muchas de las minas antiguas en los montes del Aguacate fueron reactivadas. En el año 1892 trabajaban 19 minas, 12 de ellas en los montes del Aguacate (Ulloa, 1979). La cianuración, que permite una recuperación mucho más alta del oro fue patentada en el año 1887. Tanto Reitz (1902) como Crespi (1907) mencionan molinos con hasta 20 mazos y la utilización de cianuro para lograr una recuperación del oro hasta el $90 \%$, por lo cual se podía trabajar minerales de leyes más bajas. A principios del siglo XX la "Abangares Gold Fields of Costa Rica" controló casi todas las minas de Abangares, trayendo maquinaria moderna, construyendo caminos, puentes, andariveles y líneas férreas, instalando teléfonos y luz eléctrica (Ulloa, 1979).

Durante el Primer Ciclo Minero, las minas del Aguacate emplearon a más de 400 personas y en el segundo ciclo solamente en Abangares laboraron más de 3500 . Esto es un porcentaje significativo de la población total de Costa Rica, que fue de 311 444 según el censo del año 1901 (Reitz, 1902). A principios del siglo $\mathrm{XX}$, los sueldos de los trabajadores variaba entre 3 colones (US\$1,35) por día (10 horas) para los perforadores y la mitad para los obreros corrientes (Reitz, 1902, Crespi, 1907). García (1984) menciona sueldos similares:

"Al principio del siglo XX los ademadores ganaban $ф 3,00$ por doce horas de labor, $ф 2,75$ los barreteros, $\phi 2,50$ los carreras y $\phi 2,00$ los paleros. Los mineros tenían más o menos un buen pago tomando en cuenta la época y comparándolo con el bajísimo sueldo que recibían los trabajadores de las haciendas ganaderas y de la agricultura en Guanacaste”.

\section{LA EVALUACIÓN DE LAS MINAS}

La publicación de Reitz, "The gold mines of Costa Rica”, consiste en una evaluación de diferentes minas. La introducción al artículo se basa en los relatos de don Francisco María Iglesias, quien 
redactó también las notas complementarias del artículo de Melliss (1891) y quien en el año 1877 era propietario o socio de muchas de las minas del Aguacate. Reitz era el primero en mencionar los tres distritos mineros de oro: Montes del Aguacate, Montes de Oro y Abangares, subdivisión que todavía hoy en día se mantiene. En cada distrito describe la cantidad, los espesores y los contenidos de oro en las diferentes vetas y realiza muestreos, tanto de las vetas como de las lamas que fueron dejadas por las explotaciones anteriores, para poder determinar el valor promedio de oro. En las vetas de las minas del Aguacate encontró valores que oscilan entre US\$15 hasta un máximo de US\$4000 por tonelada en las bonanzas, y también las lamas analizadas tenían valores altos, entre US\$12 y US\$79 por tonelada. Hasta los años 1970 los contenidos de oro se indicaron directamente en dólares por tonelada, porque el precio del oro no varió durante decenas de años y hubo una convertibilidad del oro en dólares (Fig. 1). Después de que el presidente Nixon eliminó la conversión oro-dólar en el año 1971 el precio del oro empezó a fluctuar y llegó a su precio más alto, de US\$850 por onza troy el 21 de enero de 1980. Como consecuencia de la liberación del precio, se indican últimamente los valores de oro en onza por tonelada o en gramos por tonelada. Sin embargo, la equivalencia a gramos y kilogramos también varió con el tiempo. Aproximadamente hasta finales del siglo XIX una onza avoirdupois equivalía a 28,35 g; posteriormente se utilizó para los metales preciosos la onza troy con $31,103 \mathrm{~g}$. La tonelada no correspondía a la tonelada métrica que utilizamos hoy en día, sino a una tonelada corta, que equivale a 2000 libras o a $907,18 \mathrm{~kg}$. Tomando en cuenta la transformación de tonelada corta en tonelada métrica y el precio del oro a principios del siglo XX (US\$20,65 por onza) podemos decir que el contenido de oro encontrado por Reitz (1902) varía entre $0,8 \mathrm{oz} / \mathrm{t}$ y $213,5 \mathrm{oz} / \mathrm{t}$ en las vetas y entre $0,64 \mathrm{oz} / \mathrm{t}$ y $4,2 \mathrm{oz} / \mathrm{t}$ en las lamas. Para tener una idea sobre la riqueza en oro de estas minas, se puede multiplicar las onzas con US\$ 589, el precio promedio del oro en los primeros seis meses del año 2006 (KITCO, 2006). Sin embargo, desde la liberación del precio, este fluctúa mucho y cinco años atrás el promedio anual era de solamente US\$ 271 por onza.

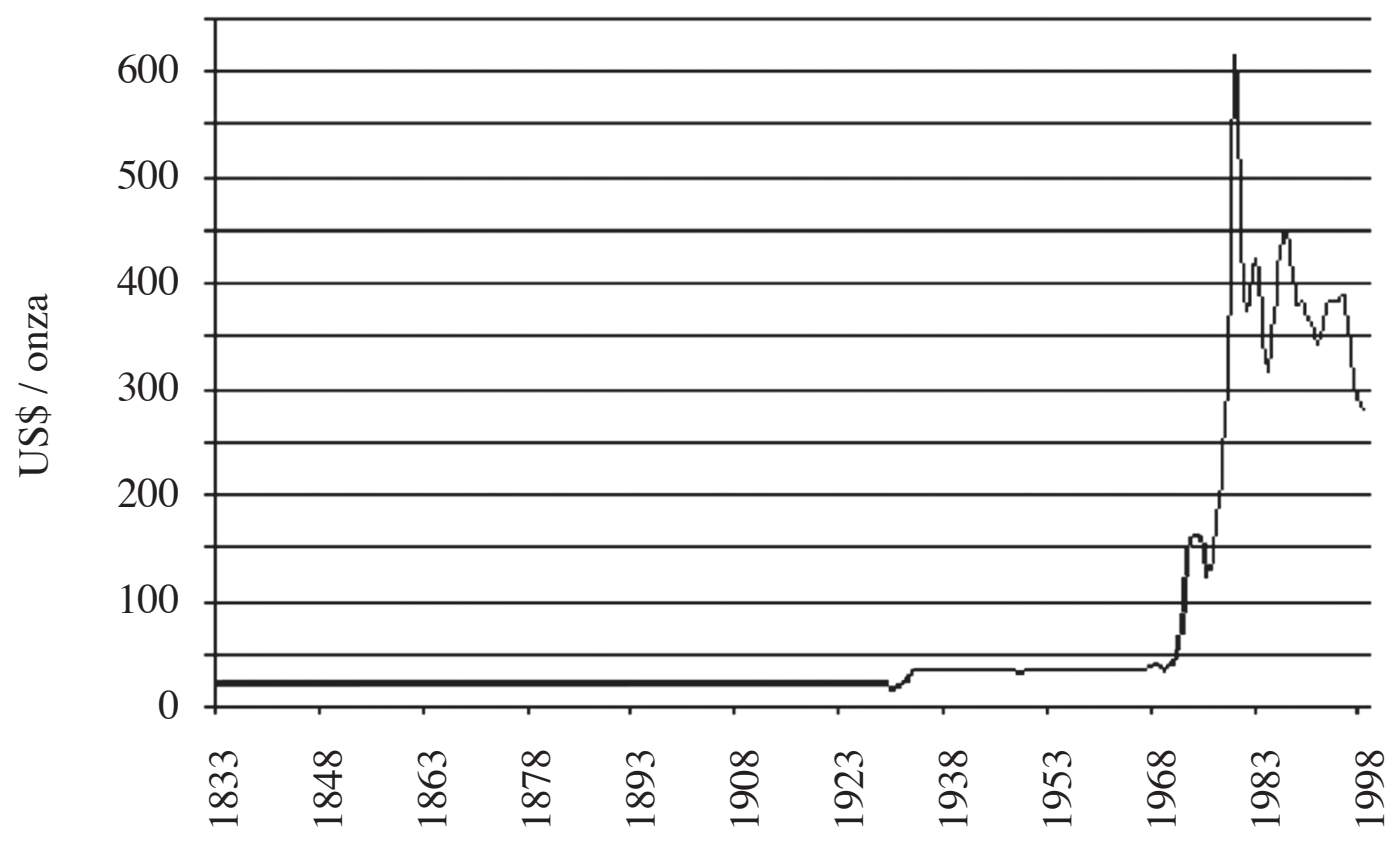

Figura 1: Evolución del precio del oro entre 1833 y 1998 (según KITCO, 2006). 


\section{Cuadro 1}

Promedio de oro en algunas minas que trabajaron a finales del siglo XX (en oz/t).

\begin{tabular}{cccccc}
\hline & Río Chiquito & Líbano & Tres Hermanos & Distrito Miramar & Sacra Familia \\
\hline OEA (1978) & -- & 0,21 & -- & 0,38 & $0,17-0,24$ \\
USGS (1987) & 0,09 & 0,84 & 0,30 & $0,21-0,64$ & 0,27 \\
\hline
\end{tabular}

Si comparamos los valores de oro que se indican en estas publicaciones con las leyes recopiladas por la OEA (1978) y el USGS (1987) en diferentes minas de oro de Costa Rica (Cuadro 1), sorprenden los valores sumamente altos que se encontraron hace más de 100 años. Esto se explica porque cuando empezó la minería del oro en Costa Rica solamente se explotaron las bonanzas y, además, los valores de oro muchas veces disminuyen con la profundidad.

Reitz menciona que la veta principal en los montes del Aguacate tiene un espesor entre 4 y 45 m y en Tres Hermanos entre 1,80 y 6 m. Estos son valores sumamente altos y poco probables. Crespi (1907) presenta un cuadro con el espesor de nueve vetas del distrito minero montes del Aguacate, donde el espesor de la veta más potente oscila entre 1,8 y $2,4 \mathrm{~m}$, todas las otras tienen menos de 2,4 m. Castillo (1997) menciona las variaciones en el espesor de muchas de las vetas de oro de Costa Rica e indica valores que generalmente oscilan entre unos pocos decímetros hasta un máximo de 19 m (en Miramar), sin embargo, la gran mayoría de los valores no exceden los $3 \mathrm{~m}$. Esta divergencia en los espesores es difícil de explicar.

\section{CONCLUSIONES}

Los tres artículos dan mucho énfasis en que las condiciones políticas y económicas en Costa Rica son favorables para operaciones lucrativas. Todos comentan que Costa Rica posee una legislación minera liberal, permite la importación libre de maquinaría y suministros y cobra pocos impuestos sobre la producción.

Reitz (1902) basa su historia de la minería en los relatos de Don Francisco María Yglesias, como lo hizo también Melliss (1891), por lo cual hay mucha coincidencia entre ambos escritos. La parte final del artículo de Crespi (1907), donde describe el clima, agua, madera y mano de obra en Costa Rica es similar a lo expuesto por Reitz (1902) sobre estos puntos, y algunos párrafos, como por ejemplo la descripción de la mano de obra, son prácticamente idénticos hasta en el monto del pago.

Crespi (1907) incluye en su artículo un mapa de Costa Rica hecho a mano alzada, copia del cual se incluye en la reproducción. Es extraño que usó un mapa con tanta deformación, a pesar de que principios del siglo XX la cartografía estaba muy adelantada lo que demuestran, por ejemplo, los mapas presentados por Reitz y von Frantzius que serán reimpresos en un próximo número de la esta revista.

El artículo "Mineral resources of Costa Rica", más que una publicación científica es una promoción de la minería en Costa Rica. No solamente habla de las muchas minas de oro y plata sino también de mineralizaciones de cobre, tungsteno, magnesio, níquel, hierro, mercurio, carbón, caolín de primera calidad, cemento, alúmina y ocres. Si bien es cierto que existen en el país manifestaciones de cobre, hierro, carbón, bauxita y ocres, que han sido explorados en el curso del siglo XX, no se conoce ninguna mineralización de tungsteno, magnesio, níquel o mercurio y la calidad de las arcillas es bastante mala. Al contrario, no se menciona el manganeso, que fue explotado en la península de Nicoya a partir del año 1916.

Otro error consiste en la altura promedio de las minas. El artículo indica 8800 pies, lo que corresponde a aproximadamente $2682 \mathrm{~m}$, altura a la cual no llega ni la cordillera de Tilarán ni los montes del Aguacate. Tanto Reitz (1902) como Crespi (1907) indican como altura promedio de las minas 1800 y 2800 pies, respectivamente.

Noticias interesantes son, que en el tiempo de la presidencia del General Tomás Guardia, se 
dictó una ley que libera a los mineros del servicio militar y se encontró una nueva versión de las minas de Tisingal.

De los tres artículos solo el de Reitz (1902) habla un poco (unas 200 palabras) sobre la geología y especialmente la mineralogía de los yacimientos. El artículo "Mineral resources of Costa Rica" solo dedica dos frases a la geología, diciendo que las rocas de Costa Rica son generalmente de origen volcánico y consisten en dioritas, pórfiros y basaltos. Cantos negros se encuentran en muchos lugares; lo mismo escribió anteriormente Reitz. Crespi (1907) menciona la geología y mineralogía en un solo párrafo, dando más énfasis a la dirección y el buzamiento de las vetas.

Llama la atención el término pórfido utilizado en las tres publicaciones. Probablemente se refiere a las lavas porfiríticas, clasificadas hoy en día como andesitas y que abundan en los montes del Aguacate y la cordillera de Tilarán. No mencionan andesitas, a pesar que este término fue introducido a principios del siglo XIX. Los cantos negros a los que hacen referencia Reitz (1902) y Anónimo (1914) son fragmentos de las vetas que presentan una pátina negra de óxidos de manganeso, a veces producto de la oxidación de la rodocrosita, y que abundan en el distrito de los montes del Aguacate.

La falta de una descripción geológica más precisa se debe a que los autores son ingenieros de minas, más dedicados a la exploración y explotación que a la geología pura. Esta falta de conocimientos geológicos se muestra mejor aún en el artículo de Melliss (1891), quien en la descripción de las minas Los Oreamuno y San Rafael dice sobre las rocas in situ solamente "La roca in situ de la masa del Aguacate es una diorita porfídica de origen metamórfico. Es generalmente homogénea y firme y no requiere maderaje en la construcción de los túneles."

Merecen, a pesar de sus incongruencias o falencias, ser reproducidos para ilustrar el conocimiento y la visión de la geología y minería en un periodo boyante de la ciencia en Costa Rica.

\section{REFERENCIAS}

ANÓNIMO, 1914: Mineral resources of Costa Rica.- Mining J. August 22, 1914: 767-768.

ARAYA POCHET, D., 1976: El segundo ciclo minero en Costa Rica (1890-1930).- 51 págs. Esc. Historía y Geografía, UCR, San José.

BERNSTEIN, P.L., 2000: El oro: Historia de una obsesión.- 586 págs. Suma de Lectores, Madrid.

CABRERA, V.M., 1924: Guanacaste.- 485 págs. Libro conmemorativo del Centenario de la incorporación del Partido de Nicoya a Costa Rica. Publ. Secretaría, Gobernación, Impr. María vda. de Linares, San José.

CASTILLO, R., 1997: Recursos minerales de Costa Rica. Génesis, distribución y potencial.- 221 págs. Ed. Universidad de Costa Rica, San José.

CRESPI, R.A., 1907: Geology and development of Aguacate mines, Costa Rica.- Mining World, November 9, 1907: 847-848.

DENYER, P., 2001: Las leyendas de las minas de Tisingal y la Estrella en Costa Rica.- Rev. Geól. America Central, 25: 49-62.

DENYER, P. \& SOTO, G.J., 2000: Análisis de los trabajos geológicos de William M. Gabb sobre Costa Rica, a la luz del paradigma geológico del siglo XIX.- Rev. Geol. Amér. Central, 23: 97-118.

ELLENBERG, L., 1987: La forma de las costas y la historia de la colonización de Costa Rica.- Serie Estudios Geográficos (Inst. Geogr. Nac. San José: 9-17.

GABB, W.M., 1875: Notes on Costa Rica geology.- Am. J. Sci. 9:198-204. 
GARCÍA, G., 1984: Las minas de Abangares: Historia de una doble explotación.- 101 págs. Ed. Univ. Costa Rica, San José.

JINESTA, R., 1938: El oro en Costa Rica.- 32 págs. Impr. Falco, San José.

KITCO, 2006: Historical charts, 1833-1999 yearly averages http://www.kitco.com/ charts/historicalgold.html [consulta 3 jul. 2006]

MELLISS, E., 1891: Las minas del monte del Aguacate y de Los Castros [traducido al español por Manuel Carazo Peralta y anotado por Francisco María Yglesias].- 20 págs.
Inst. Físico-Geogr. Nac. C.R. Tipografía Nac. San José.

OEA, 1978: Diagnóstico del sector minero.- 91 págs. República de Costa Rica, OEA, San José.

REITZ, M.F., 1902: The gold mines of Costa Rica.Eng. Mining J, August 16, 1902: 210-213.

ULLOA, F., 1979: Historia minera en Costa Rica.- 50 págs. Dir. Geol. Minas y Petróleo, San José.

USGS, 1987: Evaluación de los recursos minerales de la República de Costa Rica.- 75 págs. USGS, DGMH, UCR, Misc. Invest. Series Map I-1865. 